\title{
Sinonasal spindle cell carcinoma presenting with bilateral visual loss: A case report and review of the literature
}

\author{
TSUNG-WEI LIU ${ }^{1}$, SHIH-HAN HUNG ${ }^{2,3}$ and PO-YUEH CHEN ${ }^{1,3}$ \\ ${ }^{1}$ Department of Otolaryngology - Head and Neck Surgery, Shuang Ho Hospital, Taipei Medical University; \\ ${ }^{2}$ Department of Otolaryngology, Taipei Medical University Hospital; ${ }^{3}$ Department of Otolaryngology, \\ School of Medicine, Taipei Medical University, Taipei 110, Taiwan, R.O.C.
}

Received March 9, 2015; Accepted March 18, 2016

DOI: $10.3892 / \mathrm{ol} .2016 .4620$

\begin{abstract}
Spindle cell carcinoma (SpCC) is a rare variant of squamous cell carcinoma (SCC). SpCC of sinonasal origin is relatively rare and more aggressive than normal SCC. It most commonly involves the maxillary sinus, and rarely the sphenoid sinus. The present study reports a case of sphenoid sinus SpCC presenting with bilateral visual loss. Following endoscopic sinus decompression surgery, the patient was referred to the Oncology Department for a staging workup, and subsequently received concurrent chemoradiotherapy; however, the vision of the patient was not recovered, despite treatment. To the best of our knowledge, this is the first reported case of synchronous inverted papilloma of the sphenoid sinus and SpCC presenting with optic nerve compression.
\end{abstract}

\section{Introduction}

Spindle cell carcinoma ( $\mathrm{SpCC}$ ), also known as sarcomatoid carcinoma, pseudosarcoma and carcinosarcoma, is a rare variant of squamous cell carcinoma (SCC), accounting for $\sim 3 \%$ of all head and neck SCCs (1). It is known to be a biphasic tumor, with the epithelial component composed of poorly differentiated SCC and the mesenchymal component composed of spindle cells (2). In the head and neck region, it commonly involves the larynx, while sinonasal involvement is more rare (3). The clinical presentations of sinonasal SpCC resemble those of rhinosinusitis and there is currently no treatment protocol for sinonasal $\operatorname{SpCC}(3,4)$. The present study reports a case of synchronous inverted papilloma of the sphenoid sinus and SpCC presenting with bilateral visual loss.

Correspondence to: Dr Po-Yueh Chen, Department of Otolaryngology - Head and Neck Surgery, Shuang Ho Hospital, Taipei Medical University, 252 Wu-Hsing Street, Taipei 110, Taiwan R.O.C.

E-mail: poyueh1216@yahoo.com.tw

Key words: sinonasal, squamous cell carcinoma, spindle cell carcinoma, head and neck

\section{Case report}

A 54-year-old male patient visited the Ophthalmology Department of Shuang Ho Hospital (Taipei, Taiwan) in Febuary, 2014, complaining of left-sided vision loss for 2 weeks, and was found to have a visual acuity of 0.6 in oculus dexter (OD), and hand motion $/ 20 \mathrm{~cm}$ in the oculus sinister (OS). Fluorescence angiography revealed no obvious anomalies, and the patient was released. One week later, the patient also presented with right visual loss and returned to the Department of Ophthalmology. Visual acuity was found to have deteriorated (OD, 0.04; OS, no sensation of light). The patient reported that he had been suffering from nasal obstruction and discharge, and right frontal to parietal headache for 3 months; thus, he was referred to the Department of Otolaryngology (Shuang Ho Hospital) for further evaluation. Sinus endoscopy and computed tomography (CT; light speed VCT scanner; GE Healthcare, Milwaukee, WI, USA) scans were performed, which revealed bilateral sinusitis involving the sphenoid sinus, with nasal polyposis. The ophthalmologist scheduled an urgent head and neck CT scan, which revealed bilateral ethmoid and sphenoid sinusitis (Fig. 1). On the suspicion of sinusitis complicated with optic nerve involvement, endoscopic sinus surgery was suggested and then performed on Febuary 10, 2014. Bilateral nasal polypoid lesions were observed in the superior meatus (Fig. 2). The left sphenoid, ethmoid, frontal and right sphenoid sinuses were explored and fungus ball-like components were identified. No eschar or necrotic mucosa was found. Following surgery, the patient reported feeling that his sight had improved.

Two days after surgery, the patient again complained of visual acuity deterioration. A postoperative magnetic resonance imaging scan of the brain revealed soft-tissue density filling the sphenoid sinus. The bilateral optic nerves at the intraconal region were found to be intact; however, optic nerve involvement by the sphenoid sinusitis was suspected near the bilateral optic canals and before the optic chiasm (Fig. 3).

The pathological finding revealed features of inverted papilloma in a few sections of respiratory mucosa and also a few pleomorphic spindle cells blending with the dysplastic squamous cells. Synchronous inverted papilloma of the sphenoid sinus and SpCC were diagnosed. Hematoxylin and eosin staining showed the tumor to be composed of pleomorphic 


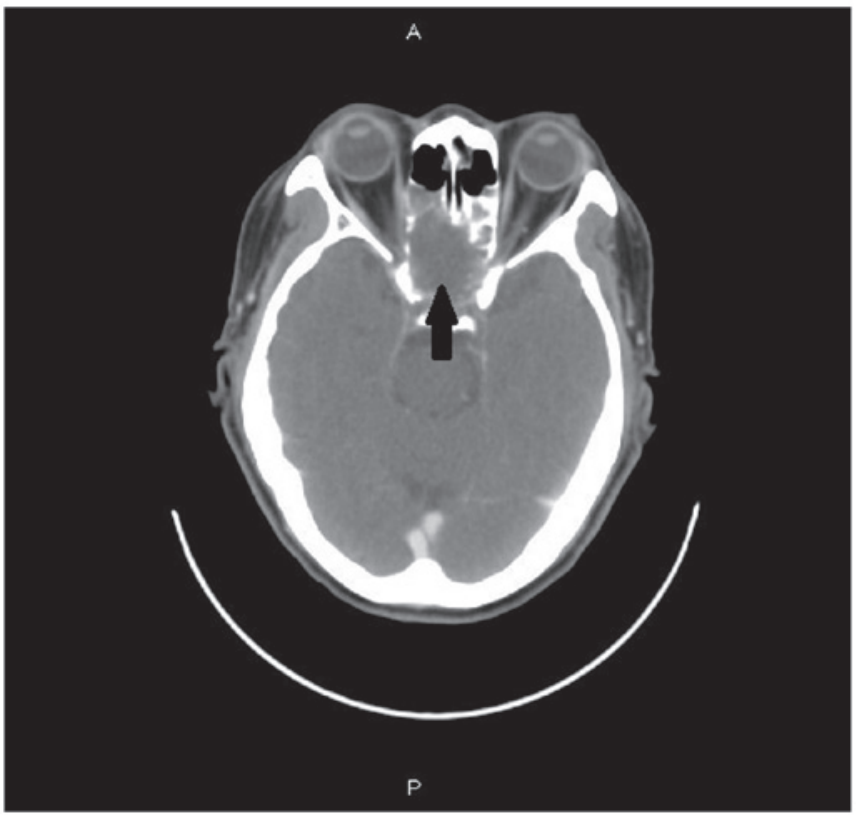

Figure 1. Computed tomography of the sinus revealed bilateral sinusitis involving the ethmoid and the sphenoid sinuses (arrow).

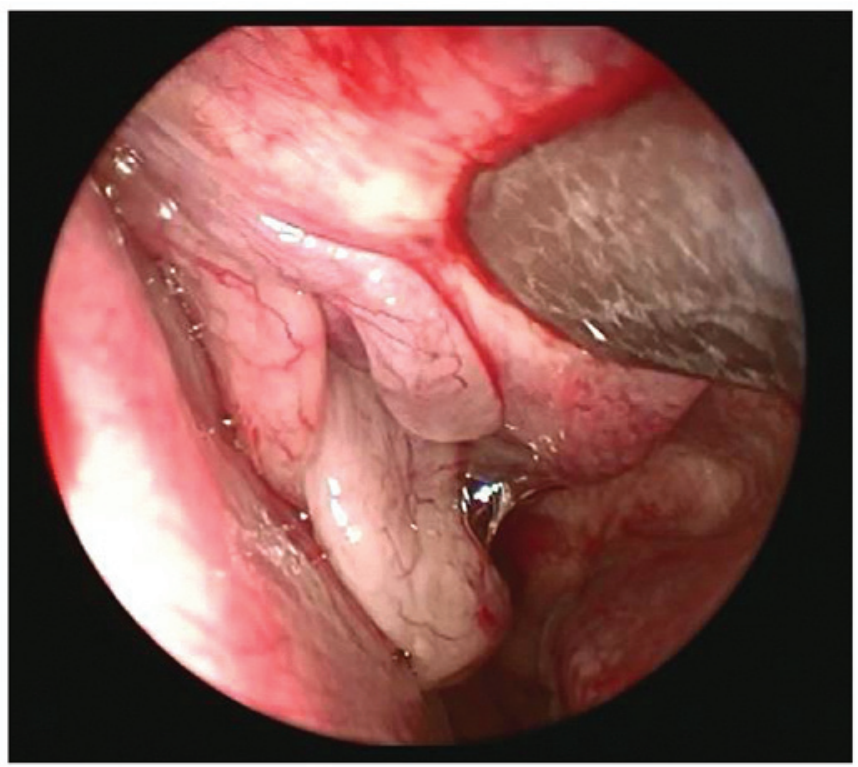

Figure 2. Nasal endoscopy revealing bilateral nasal polypoid lesions in the superior meatus.

spindle cells intermixed with dysplastic squamous cells (Fig. 4). An immunohistochemical study was performed with the use of the Dako EnVision method (Dako, Carpinteria, CA, USA), and the spindle cells showed focal positive immunoreactivity for pan cytokeratin (monoclonal mouse anti-human antibody; catalog no., 760-2135; ready to use; Ventana Medical Systems, Inc., Tuscon, AZ, USA) (Fig. 5). No fungus was identified.

Ceftriaxone (1,000 mg by intravenous drip every $8 \mathrm{~h}$ ) was administered for 7 days and endoscopic sinus surgical decompression was performed again. The patient was then referred to the Department of Oncology (Shuang Ho Hospital) and following a staging workup, received concurrent chemoradiotherapy with $50 \mathrm{mg}$ weekly cisplatin and $70 \mathrm{~Gy}$ radiotherapy in

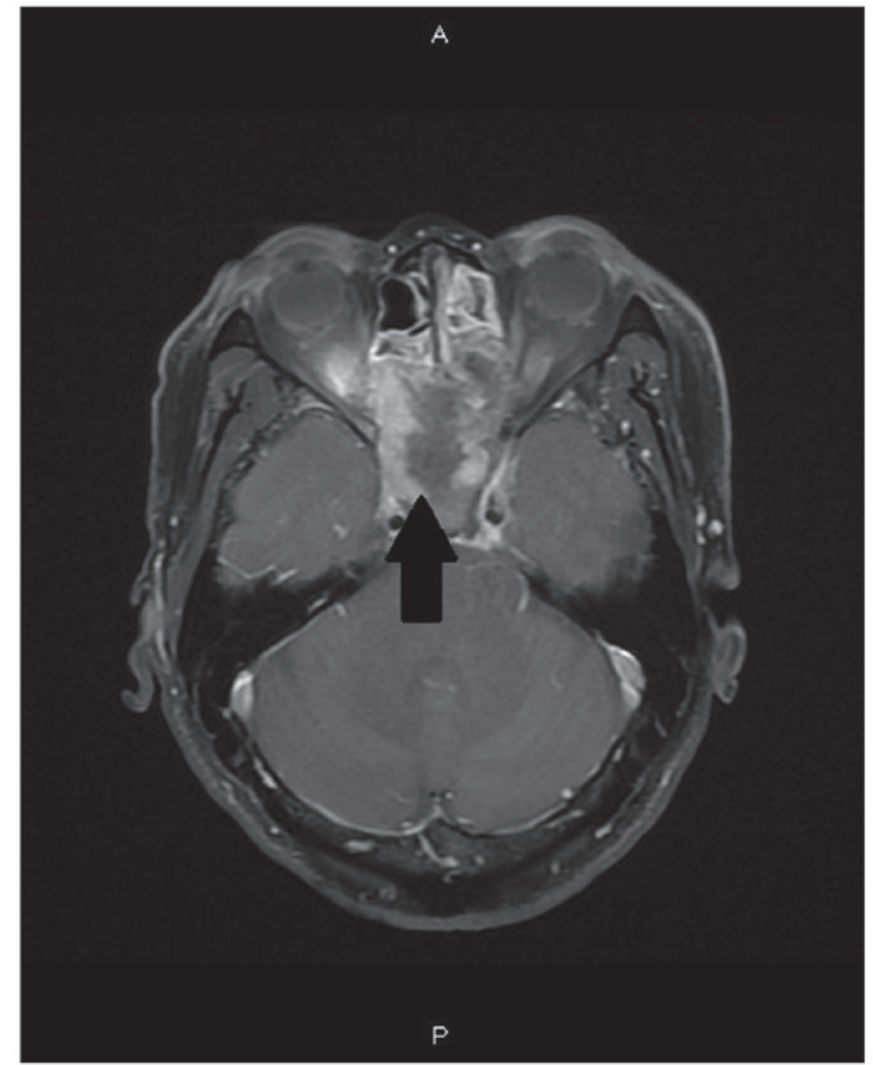

Figure 3. Postoperative magnetic resonance imaging showing that the sphenoid sinus was filled with soft-tissue density. Bilateral optic nerve involvement by the sphenoid sinusitis was suspected near the bilateral optic canals and before the optic chiasm (arrow).

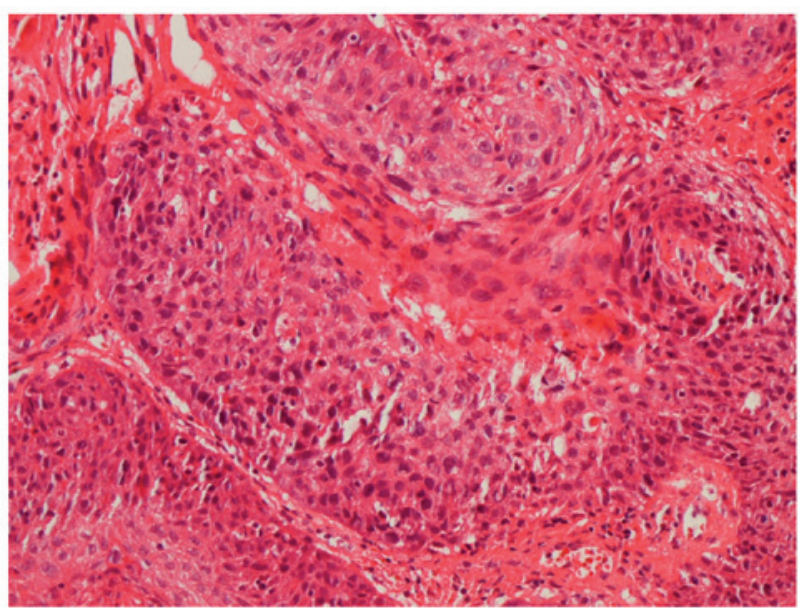

Figure 4. Hematoxylin and eosin staining showing that the tumor was composed of pleomorphic spindle cells mixed with dysplastic squamous cells (magnification, x100).

35 fractions. The left eye was unresponsive to light following 2 months of concurrent chemoradiotherapy, and the patient succumbed to bone and liver metastasis 12 months later.

\section{Discussion}

SpCC, also known as sarcomatoid carcinoma, pseudosarcoma, and carcinosarcoma, is an unusual variant of SCC that 
Table I. Locations of spindle cell carcinoma.

\begin{tabular}{|c|c|c|c|}
\hline First author/s, year & Cases, $\mathrm{n}$ & Location $^{\mathrm{a}}$ & Refs. \\
\hline Howell et al, 1978 & 13 & Sinonasal cavity & $(6)$ \\
\hline Leventon and Evans, 1981 & 1 & Maxillary sinus & $(7)$ \\
\hline Benninger et al, 1992 & 2 & Maxillary sinus & $(8)$ \\
\hline Asbury et al, 1992 & 1 & Maxillary sinus & $(9)$ \\
\hline Berthelet et al, 1994 & 1 & Nasal cavity & $(10)$ \\
\hline Ahluwalia et al, 1996 & 1 & Nasal cavity & $(11)$ \\
\hline Mills et al, 1997 & 18 & Sinonasal cavity & $(12)$ \\
\hline Sadaba et al, 2006 & 1 & Maxillary sinus & $(13)$ \\
\hline Howard et al, 2007 & 1 & Maxillary sinus & $(4)$ \\
\hline Kumar et al, 2008 & 1 & Maxillary sinus & (14) \\
\hline Minton and Goyal, 2009 & 1 & Maxillary sinus & $(15)$ \\
\hline Viswanathan et al, 2010 & 6 & Maxillary sinus & $(3)$ \\
\hline Doshi et al, 2010 & 19 & Sinonasal cavity & $(16)$ \\
\hline Terada, 2011 & 1 & Maxillary sinus and nasal cavity & $(17)$ \\
\hline Terada and Kawasaki, 2011 & 1 & Nasal cavity & $(18)$ \\
\hline Gupta et al, 2011 & 1 & Nasal cavity & $(2)$ \\
\hline
\end{tabular}

Inverted papilloma was not present in any case.

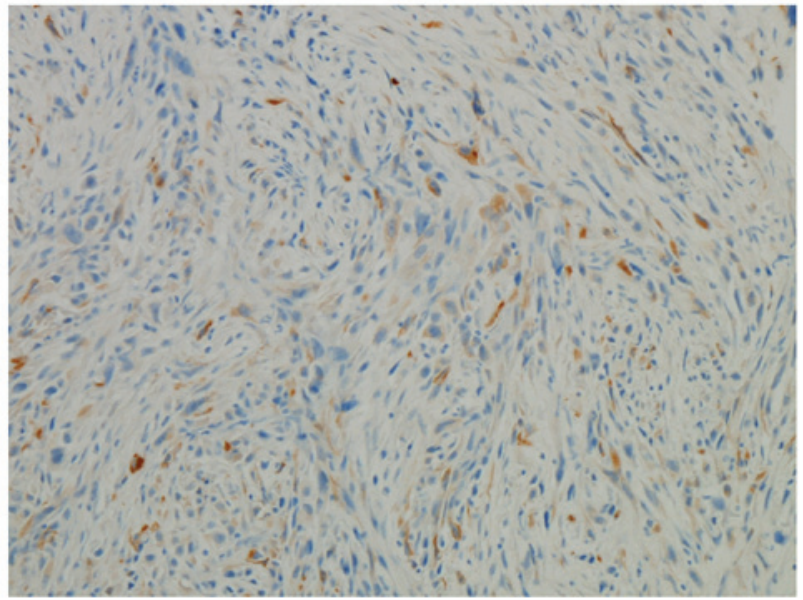

Figure 5. Immunohistochemical staining showing focal positive immunoreactivity of the spindle cells for pan cytokeratin (magnification, $\mathrm{x} 200$ ).

accounts for $3 \%$ of all head and neck SCCs (1). It has been proposed that this biphasic tumor may arise from conventional SCC by sarcomatous transformation $(2,3)$. SpCC most commonly occurs in the fifth and sixth decades of life, and is associated with a male preponderance. Risk factors for SpCC include smoking, alcohol consumption and previous radiotherapy $(2,3,5)$. Among the organs in the head and neck region, the larynx is the most common site of involvement, followed by the oral cavity (1). In the sinonasal areas, maxillary sinus involvement is commonly observed, while sphenoid sinus involvement is considered rare (Table I) (2-4,6-18).

Viswanathan et al (3) reported 103 cases of head and neck SpCC, and found that $46.6 \%$ presented with obvious epithelial differentiation and $33 \%$ with epithelial differentiation at the immunohistochemical level, while $20.4 \%$ displayed no evidence of either. The majority of patients with sinonasal SpCC present with excessive tearing, nasal obstruction, facial swelling and numbness, and nasal purulence (2). Orbital symptoms are less commonly observed $(1,2)$. In addition, to the best of our knowledge, only one case of sinus SpCC presenting with orbital apex syndrome has been reported (13). The symptoms of the 45-year-old male patient included left eyelid swelling, diplopia and proptosis. Visual acuity was initially 20/20. Imaging scans revealed that a left maxillary sinus mass had eroded the floor of the left orbit and extended to the left retrobulbar region, apex of the orbit and optic chiasm. Orbital involvement appears to be associated with a poor prognosis, as the left eye of this patient was unresponsive to light following 2 months of concurrent chemoradiotherapy, and he succumbed to bone and liver metastasis 12 months later (13).

In contrast to the aforementioned case, the patient in the present study presented with no obvious signs of gross tumor invasion into the orbital cavity, despite also initially complaining of vision loss. Among all sphenoid lesions with ocular manifestations, benign sphenoid mucoceles are the most commonly reported, with the majority of the ocular manifestations, including visual acuity, recovering following lesion resolution $(19,20)$; however, one case of extramedullar plasmacytoma in the sphenoid sinus presenting with optic nerve compression failed to regain visual acuity following pressure relief by surgery (21).

Following a literature review of sinonasal SpCC cases (Table I) (2-4,6-18), it was concluded that there does not appear to be an association between the lesion and the presence of sinonasal inverted papilloma; however, since the inverted papilloma can potentially transform into SCC, it is likely that it can also transform into $\mathrm{SpCC}$, as it is a variant of SCC. We believe that the chronicity of inverted papilloma is associated with the induction of SpCC transformation. To the best of our 
knowledge, this is the first reported case of inverted papilloma of the synchronous sphenoid sinus and SpCC presenting with optic nerve compression.

In conclusion, $\mathrm{SpCC}$ of sinonasal origin is relatively rare and clinically aggressive. In the majority of cases, maxillary sinus involvement was observed, while sphenoid sinus involvement was more rare. The presentation of orbital symptoms has been associated with a relatively poor prognosis and, therefore, the clinical management of such patients should be more aggressive. The value of surgical decompression in these cases remains unclear.

\section{References}

1. Thompson LDR: Squamous cell carcinoma variants of the head and neck. Curr Diagn Pathol 9: 384-396, 2003.

2. Gupta S, Santoriello D, Wieczorek R and De Lacure MD: Spindle cell carcinoma of the nasal cavity. Rare Tumors 5: 10, 2013.

3. Viswanathan S, Rahman K, Pallavi S, Sachin J, Patil A, Chaturvedi P, D'Cruz A, Agarwal J and Kane SV: Sarcomatoid (spindle cell) carcinoma of the head and neck mucosal region: A clinicopathologic review of 103 cases from a tertiary referral cancer centre. Head Neck Pathol 4: 265-275, 2010.

4. Howard SN, Bond WR, Hong IS and Foss RD: Right maxillary sinus sarcomatoid carcinoma (sarcomatoidspindle cell carcinoma). Otolaryngol Head Neck Surg 137: 355-357, 2007.

5. Chang NJ, Kao DS, Lee LY, Chang JW, Hou MM, Lam WL and Cheng MH: Sarcomatoid carcinoma in head and neck: A review of 30 years of experience-clinical outcomes and reconstructive results. Ann Plast Surg 71 (Suppl 1): S1-S7, 2013.

6. Howell JH, Hyams VJ and Sprinkle PM: Spindle cell carcinoma of the nose and paranasal sinuses. Surg Forum 29: 565-568, 1978

7. Leventon GS and Evans HL: Sarcomatoid squamous cell carcinoma of the mucous membranes of the head and neck: A clinicopathologic study of 20 cases. Cancer 48: 994-1003, 1981.

8. Benninger MS, Kraus D, Sebek B, Tucker HM and Lavertu P Head and neck spindle cell carcinoma: An evaluation of current management. Cleve Clin J Med 59: 479-482, 1992.
9. Asbury L, Candelaria S, Rudak F, Stutzman CD and Lake DE: High dose rate treatment of a maxillary sarcomatoid carcinoma: A case report. Med Dosim 17: 129-133, 1992.

10. Berthelet E, Shenouda G, Black MJ, Picariello M and Rochon L: Sarcomatoid carcinoma of the head and neck. Am J Surg 168: 455-458, 1994.

11. Ahluwalia H, Gupta SC and Gupta SC: Pathology in focus. Spindle-cell carcinoma of the nasal septum. J Laryngol Otol 110: 284-287, 1996.

12. Mills S, Gaffey M and Frierson Jr H (eds); Armed Forces Institute Of Pathology: Spindle cell carcinoma. In: Tumors of the Upper Aerodigestive Tract and Ear [Atlas Of Tumor Pathology (AFIP) 3rd Series]. Armed Forces Institute of Pathology, Washington, DC, pp76-80, 1997.

13. Sadaba LM, Garcia-Layana A, Garcia-Gomez PJ and Salinas-Alaman A: Sarcomatoid carcinoma and orbital apex syndrome. Eur J Ophthalmol 16: 608-610, 2006.

14. Kumar M, Bahl A, Sharma DN, Agarwal S, Halanaik D, Kumar R and Rath GK: Sarcomatoid squamous cell carcinoma of uterine cervix: Pathology, imaging and treatment. J Cancer Res Ther 4: 39-41, 2008.

15. Minton TJ and Goyal P: Endoscopic treatment of a maxillary sinus spindle cell carcinoma. J Otolaryngol Head Neck Surg 38: E45-E50, 2009.

16. Doshi DV, Tripathi U, Dave RI, Pandya SJ, Shukla HK and Parikh BC: Rare tumors of sinonasal track. Indian J Otolaryngol Head Neck Surg 62: 111-117, 2010

17. Terada T: Pure sarcomatoid carcinoma of maxillary sinus and nasal cavity simulating malignant fibrous histiocytoma. Am J Clin Pathol 135: 128-131, 2011 .

18. Terada T and Kawasaki T: Spindle cell carcinoma of the nasal cavity. Int J Clin Oncol 16: 165-168, 2011.

19. Hejazi N, Witzmann A and Hassler W: Ocular manifestations of sphenoid mucoceles: Clinical features and neurosurgical management of three cases and review of the literature. Surg Neurol 56: 338-343, 2001.

20. Yoon TM, Kim K and Lim SC: Visual outcome after endoscopic decompression surgery for sphenoid sinus disease: How we do it. Clin Otolaryngol 33: 480-484, 2008.

21. Ozdemir S, Tarkan O, Tuncer U, Sürmelioğlu O, Doğrusöz M and Ergin M: A case of extramedullary plasmacytoma in the sphenoid sinus with unilateral loss of vision. J Craniomaxillofac Surg 41: 140-143, 2013. 